\title{
PERCEPCIONES DE ALUMNOS Y DOCENTES DE 5. Y 6. DE EDUCACIÓN PRIMARIA SOBRE LA MODALIDAD DE EDUCACIÓN A DISTANCIA IMPLANTADA TEMPORALMENTE EN ESPAÑA POR COVID-19
}

\author{
Perceptions of Students and Teachers of 5th and Gth Grade \\ of Primary Education about the Distance Education \\ Modality Implemented in Spain, Temporarily, because \\ of Covid-19
}

\author{
Arántzazu DE LAS MORENAS MARTíN \\ Universidad Nacional de Educación a Distancia (UNED). España \\ Correo-e: adelasmor2@alumno.uned.es \\ bttps://orcid.org/0000-0003-1266-3496
}

Recibido: 03/12/2020; Aceptado: 15/12/2020; Publicado: 27/12/2020

Ref. Bibl. ARÁNTZAZU DE LAS MORENAS MARTÍN. Percepciones de alumnos y docentes de $5 .^{\circ}$ y $6 .^{\circ}$ de Educación Primaria sobre la modalidad de educación a distancia implantada temporalmente en España por COVID-19. Enseñanza $\varepsilon$ Teaching, 38, 2-2020, 157-175.

RESUMEN: El objetivo de este estudio ha sido analizar la modalidad de educación a distancia implantada en España durante el periodo de confinamiento por Covid-19 a través de la percepción de alumnos y docentes. Se analiza una muestra de 99 estudiantes y 31 docentes de $5 .^{\circ}$ y $6 .^{\circ}$ curso de Educación Primaria de centros públicos, concertados y no concertados en el municipio de Alcalá de Henares (Comunidad de Madrid). La investigación alterna la metodología cuantitativa y cualitativa complementando el análisis de cuestionarios, entrevistas y preguntas abiertas como instrumentos principales del estudio. Los resultados generales muestran que los alumnos han continuado trabajando en sus tareas escolares durante 
PERCEPCIONES DE ALUMNOS Y DOCENTES DE $5 .^{\circ} \mathrm{Y} 60^{\circ}$ DE EDUCACIÓN PRIMARIA SOBRE LA MODALIDAD DE EDUCACIÓN A DISTANCIA IMPLANTADA TEMPORALMENTE EN ESPAÑA POR COVID-19

el confinamiento, así como manteniendo contacto con sus maestros/as, siendo los dispositivos digitales más recurridos para la búsqueda de información, la revisión del correo electrónico y la entrega y recepción de tareas, correcciones y puntuaciones el smartphone y el ordenador portátil en el caso de los estudiantes de centros públicos y el portátil y la tablet en el caso de los concertados y no concertados. Los docentes, por su parte, coinciden en afirmar que los alumnos no estaban preparados para este cambio repentino en la modalidad educativa, apuntando como dificultades detectadas la competencia digital del alumnado, la escasez de medios y la falta de proximidad que complica el feedback docente-discente.

Palabras clave: TIC; dispositivos digitales; aprendizaje a distancia; confinamiento; Educación Primaria.

SUMMARY: The aim of this study has been to analyze the distance education modality implemented in Spain during the period of confinement by Covid-19, through the perception of students and teachers. A sample of 99 students and 31 teachers of 5th and 6th grade of Primary Education from public, subsidized and non-subsidized schools in the municipality of Alcala de Henares (Community of Madrid) is analyzed. The research alternates the quantitative and qualitative methodology, complementing the analysis of questionnaires, interviews and open questions as the main instruments of the study. The general results show that students have continued to work on their homework during confinement, as well as maintaining contact with their teachers, being the most frequently used digital devices for searching for information, checking email, and delivering and receiving of homework, corrections and scores, the Smartphone and Laptop in the case of students from public schools, and the Laptop and Tablet in the case of concerted and non-concerted students. Teachers, for their part, agree that the students were not prepared for this sudden change in the educational modality, pointing out as difficulties detected the digital competence of the students, the shortage of media and the lack of proximity that complicates teacher-student feedback.

Key words: ICT; digital devices; distance learning; confinement; Primary Education.

\section{INTRODUCCIÓN}

La incorporación de las Tecnologías de la Información y la Comunicación a la Sociedad, en especial al ámbito educativo, ha ido adquiriendo una gran importancia con el paso de los años, evolucionando hasta el punto de saltar de la "posibilidad" a la "necesidad», convirtiéndose en una herramienta de trabajo básica tanto para estudiantes como para alumnos. La escuela se configura como el escenario perfecto desde el que empezar a trabajar la competencia digital, a través de la alfabetización, la renovación del material didáctico o el empleo de nuevas herramientas de trabajo digitales. 
PERCEPCIONES DE ALUMNOS Y DOCENTES DE $5 .^{\circ} \mathrm{Y}$ 6. ${ }^{\circ}$ DE EDUCACIÓN PRIMARIA SOBRE LA MODALIDAD DE EDUCACIÓN A DISTANCIA IMPLANTADA TEMPORALMENTE EN ESPAÑA POR COVID-19

Cada vez son más autores que plantean estudios sobre la posibilidad de implantar dispositivos digitales para uso didáctico, proponiendo, además, modelos de integración en los procesos de enseñanza-aprendizaje de materiales didácticos digitales, persiguiendo, en todo momento, el trabajo autónomo, la motivación y la mejora del rendimiento académico de los alumnos.

Estudios como el de Sáez et al. (2011) demuestran que el uso de dispositivos móviles representa una atractiva herramienta de trabajo en el ámbito educativo y que el grado de disposición de los alumnos hacia el manejo de estos dispositivos y de satisfacción con las actividades diseñadas es alto. Y documentos como el de Camacho y Esteve (2018) ponen de manifiesto la actitud favorable de los alumnos hacia las TIC, tanto a nivel personal como en su aplicación en la educación (percibiéndose competentes en la utilización de la tecnología móvil), y la amplia variedad didáctica de posibilidades que ofrecen las tabletas digitales, tanto a nivel de contenido como de estrategias metodológicas o aplicaciones móviles utilizadas.

Aun así, la incorporación de los dispositivos digitales al aula se encuentra supeditada a aspectos culturales, políticos o económicos. Fernández (2016) alude a Serrano y Martínez (2003) para explicar que cada día la brecha digital -entendida como la separación entre personas que utilizan las TIC como parte rutinaria de la vida cotidiana y las que no tienen acceso a las mismas- resulta menos evidente dado que a través de los dispositivos móviles casi todo el mundo puede acceder al conocimiento de las TIC sin tener que realizar una gran inversión en comprar un ordenador.

Sin embargo, son muchos los centros educativos que han «incorporado» en sus aulas las TIC como dispositivos "Complementarios" $\mathrm{y}$ "aislados" de todo proceso educativo. Pero ino ha dado el confinamiento por Covid-19 un vuelco a todo lo concebido hasta el momento, atribuyendo a las Tecnologías de la Información y la Comunicación y a los dispositivos digitales un papel nunca antes pensado? ¿No es cierto que jamás imaginamos que la continuidad de la educación podía depender, de forma temporal, de modelos educativos digitales que nos resultaran, en ciertos casos, inalcanzables, a pesar de vivir en plena Era Digital? Feito (2020) hacía alusión al respecto con las siguientes palabras:

La crisis provocada por la COVID-19 ha situado a la escuela en un escenario inédito. De un día para otro, la enseñanza -cuando esto ha sido posible- ha pasado de ser presencial a virtual. [...] Profesores, alumnos y padres y madres están accediendo a contenidos didácticos que ponen de manifiesto que es posible aprender de otra manera, de un modo más autónomo. [...].

Fernández y Vergara (2020) reflejan la necesidad de un cambio del paradigma educativo señalando como datos que el $70 \%$ de los puestos de trabajo del futuro no existen; que el $50 \%$ de los nuevos puestos de trabajo quedan sin cubrir por no encontrar profesionales con habilidades digitales necesarias, y que entre el $50 \%$ y el 80 \% de los alumnos de la Unión Europea aún no emplean métodos didácticos digitales, como libros de texto, realidad virtual, podcasts o gamificaciones a lo largo de su etapa educativa. 

DE EDUCACIÓN A DISTANCIA IMPLANTADA TEMPORALMENTE EN ESPAÑA POR COVID-19

Resulta innegable que la pandemia nos ha permitido reflexionar sobre la educación que tenemos y sobre el aprovechamiento que hacemos de la gran variedad de herramientas y recursos didácticos que tenemos a nuestro alcance.

Según Servimedia (22 de mayo de 2020), el estudio titulado «El impacto de las pantallas en la vida familiar durante el confinamiento", llevado a cabo por Empantallados y GAD3, con el apoyo de la Comisión Europea, uno de cada dos padres y madres compraron algún dispositivo durante el periodo mencionado: un $49 \%$ para las clases de sus hijos, un $29 \%$ para su trabajo y un $20 \%$ para ocio digital. Pero, además, los resultados de este estudio apuntan que, de lunes a viernes, los más pequeños comenzaron a utilizar las pantallas casi cuatro horas diarias (un $76 \%$ más que antes del confinamiento). Asimismo, el $85 \%$ de los padres reconocen en el estudio que los dispositivos han creado nuevas oportunidades para hacer cosas con sus hijos (como ver películas o jugar juntos) y más del $75 \%$ afirma que suponen una ventana al exterior, pues gracias a ello ha sido posible la comunicación con familiares y amigos.

Ecoaula.es (13 de mayo de 2020) recoge en su artículo la confianza de los padres españoles en la tecnología para el desarrollo profesional y educativo de sus hijos. Así, nueve de cada diez padres opinan que la tecnología que sus hijos emplean les beneficiará en sus futuras carreras profesionales (a pesar de que a siete de cada diez les preocupa el tiempo empleado frente a las pantallas).

\section{METAS}

\subsection{Objetivo general}

Estudiar la modalidad de educación a distancia implantada durante el confinamiento por Covid-19 en centros públicos, concertados y no concertados del municipio de Alcalá de Henares (Comunidad de Madrid) bajo la percepción de alumnos y docentes de quinto y sexto curso de Educación Primaria, a través de una investigación que complementa la metodología cuantitativa y cualitativa.

\subsection{Objetivos específicos}

- Analizar el uso didáctico que los alumnos han hecho de los dispositivos digitales: smartphone, tablet y ordenador, durante el confinamiento atendiendo al contacto mantenido con sus docentes, sus posibilidades de acceso a Internet o la titularidad de los centros en los que se encuentran matriculados.

- Examinar la labor del personal docente durante el confinamiento a partir de la carga lectiva impuesta, las herramientas de trabajo empleadas, la comunicación establecida con alumnos, familias y resto de profesores o la titularidad de los centros educativos en los que trabajan. 
PERCEPCIONES DE ALUMNOS Y DOCENTES DE $5 .^{\circ} \mathrm{Y} 6 .^{\circ}$ DE EDUCACIÓN PRIMARIA SOBRE LA MODALIDAD DE EDUCACIÓN A DISTANCIA IMPLANTADA TEMPORALMENTE EN ESPAÑA POR COVID-19

- Contrastar las experiencias personales de dos maestras y un maestro en su ocupación docente durante el confinamiento, teniendo en cuenta sus vivencias personales en el proceso de adaptación y desarrollo de la nueva modalidad impuesta temporalmente.

\section{MÉTODO}

Clasificamos este artículo como un tipo de investigación en el que se alterna el estudio cuantitativo de cuestionarios aplicados a alumnos y docentes con el cualitativo a través del análisis de respuestas a preguntas abiertas en los mencionados cuestionarios y de transcripciones a entrevistas realizadas a maestros y maestras. El estudio está enfocado a la recolección de datos sobre el proceso seguido en Educación Primaria en la modalidad de enseñanza telemática a distancia implantada temporalmente, en toda España, durante el periodo de confinamiento por Covid19. Acotamos la investigación a centros públicos, concertados y no concertados del municipio de Alcalá de Henares (Comunidad de Madrid), estudiando las percepciones de alumnos y docentes de tercer ciclo de la etapa mencionada (cursos $5 .^{\circ}$ y $6 .^{\circ}$ de Educación Primaria), abordando diversas variables, más adelante especificadas.

Para obtener las experiencias e impresiones de los alumnos y docentes empleamos como instrumentos de análisis dos cuestionarios (elaborados a través de "Google Forms" y difundidos por diversas redes sociales) y entrevistas semiestructuradas personales a tres docentes de tercer ciclo de Primaria en Alcalá de Henares. Los datos obtenidos de las respuestas cerradas a los cuestionarios han sido recogidos en dos bases de datos diferenciadas (una con la información de los formularios de alumnos y otra con la de docentes), utilizando el programa estadístico SPSS y conformando, así, nuestro estudio cuantitativo del artículo. Por su parte, las entrevistas han sido transcritas y analizadas a través de un sistema de categorización mixto, junto con el contenido textual de las respuestas abiertas de los cuestionarios a docentes y discentes.

Se realizó un registro de los centros de diversa titularidad en el municipio de Alcalá de Henares y se utilizó el correo electrónico, diversas redes sociales (Facebook, Twitter e Instagram) y sistemas de mensajería instantánea (WhatsApp) para emprender la difusión a docentes y estudiantes. Entre los colegios registrados destacamos: Miguel Hernández, Juan de Austria, La Garena, Infanta Catalina, Santos Niños, Daoiz y Velarde y Cristóbal Colón (centros públicos); Sagrado Corazón de Jesús, San Felipe Neri, Gredos San Diego, Alborada, San Gabriel y Lope de Vega (centros concertados), y Santo Tomás de Aquino (centro no concertado). Tras la selección final se obtuvieron un total de 99 respuestas de discentes válidas y 31 de docentes. El contacto con los tres docentes que participaron en nuestro estudio a través de entrevistas individuales fue vía telefónica y las entrevistas tuvieron una duración estimada de unos 30 minutos. Destacamos que dos de nuestros docentes (un maestro y una maestra) ejercen su labor docente en instituciones de titularidad concertada y una en una institución pública. Asimismo, señalamos que tratamos 
PERCEPCIONES DE ALUMNOS Y DOCENTES DE $5 .^{\circ} \mathrm{Y} 60^{\circ}$ DE EDUCACIÓN PRIMARIA SOBRE LA MODALIDAD DE EDUCACIÓN A DISTANCIA IMPLANTADA TEMPORALMENTE EN ESPAÑA POR COVID-19

de obtener la participación de maestros de variada edad, logrando las edades de 26, 52 y 60. Las llamadas telefónicas con los maestros fueron grabadas, bajo previo consentimiento, para su posterior transcripción y análisis de contenido a través de tablas y según el, ya mencionado, sistema categorial mixto.

\section{RESUltados}

Partiendo de la siguiente hipótesis: La enseñanza telemática implantada en España durante la pandemia por Covid-19 ha incrementado el uso didáctico de dispositivos digitales y herramientas virtuales permitiendo el trabajo de contenidos desde casa y facilitando, así, los procesos de enseñanza-aprendizaje durante el periodo de confinamiento. Trataremos, con este estudio, de dar respuesta a las siguientes cuestiones:

\subsection{Atendiendo a las percepciones de alumnos de $5 .^{\circ}$ y $6 .^{\circ}$ de Educación Primaria de centros públicos, concertados y no concertados de Alcalá de Henares}

Contamos con una muestra de 99 alumnos de $5 .^{\circ}$ y $6 .^{\circ}$ de Educación Primaria, escolarizados en centros públicos, concertados y no concertados de Alcalá de Henares, que accedieron a participar en nuestro estudio. Se comprueba un porcentaje equilibrado en cuanto a "curso" (un $47,5 \%$ alumnos de $5 .^{\circ}$ de E. P. y un $52,5 \%$ de $6 .^{\circ}$ de E. P.) y "titularidad pública y concertada", perteneciendo un $53,5 \%$ de nuestro alumnado a la primera y un $42,5 \%$ a la segunda (el porcentaje de participación en nuestro estudio de la no concertada se redujo a un escaso $4 \%$ ).

Se comprueba una asociación estadísticamente significativa $(C=0,305$ y $\mathrm{p}=$ $0,037<0,05)$ entre la titularidad de los centros en los que se encuentran escolarizados los alumnos que participaron en el estudio y el contacto con sus docentes durante el periodo de confinamiento, pues, a pesar de que el porcentaje mayoritario en las tres titularidades fue aquel que señaló "haber estado en contacto con su tutor/a y profesores de otras asignaturas», se observa una diferencia porcentual significativa entre ellas (siendo de un $52,83 \%$ en la enseñanza pública, de un $83,33 \%$ en la concertada y de un $75 \%$ en la no concertada). De igual forma, el porcentaje de alumnos de centros públicos que solo estuvo en contacto con los tutores durante el confinamiento fue de un 45,29\% (frente a un 16,67\% de la concertada y a un $25 \%$ de la no concertada).

En cuanto al acceso a Internet, resaltamos que el $100 \%$ de nuestros alumnos manifestaron disponer de conexión a Red Wifi y un 85 \% declaró contar con un dispositivo propio para realizar las tareas y/o comunicarse con el profesor durante el confinamiento (el $15 \%$ que reveló no disponer de un dispositivo propio quedó distribuido en un $10 \%$ de alumnos de centros públicos y un $5 \%$ de centros concertados, pues ningún alumno de la no concertada señaló no disponer de dispositivo propio). 
PERCEPCIONES DE ALUMNOS Y DOCENTES DE $5 .^{\circ} \mathrm{Y} 6 .^{\circ}$ DE EDUCACIÓN PRIMARIA SOBRE LA MODALIDAD DE EDUCACIÓN A DISTANCIA IMPLANTADA TEMPORALMENTE EN ESPAÑA POR COVID-19

A la pregunta: ¿Te resulta fácil utilizar el dispositivo para realizar las tareas y/o comunicarte con el profesor? Un 69,7 \% respondió de manera afirmativa, un 28,3\% confesó necesitar ayuda de algún familiar y un escaso 2 \% señaló resultarle muy complicado. La facilidad de los alumnos para manejar los dispositivos didácticos fue cruzada, en este estudio, con el curso académico de los mismos y los resultados porcentuales muestran que a un $63,8 \%$ de los alumnos de $5 .^{\circ}$ de Primaria le resultó sencillo (porcentaje que asciende al $75 \%$ en $6 .^{\circ}$ curso), un 31,9\% necesitó ayuda (porcentaje que desciende al $25 \%$ en $6^{\circ}$ ) y a un $4,3 \%$ le pareció muy complicado (ningún alumno de $6 .^{\circ}$ encontró demasiado complicado su manejo). No obstante, el cálculo del "Coeficiente de Contingencia» $(\mathrm{C}=0,173)$ y la prueba "Chi-Cuadrado" no resaltan una asociación estadísticamente significativa ( $\mathrm{p}=0,215$ $>0,05$ ) entre ambas variables (curso y facilidad de manejo de los dispositivos con fines didácticos).

El 52,5 \% del alumnado que respondió a nuestro cuestionario había utilizado su dispositivo para realizar las tareas y/o comunicarse con el profesor antes del confinamiento ( $41,5 \%$ en la titularidad pública, $64,3 \%$ en la concertada y $75 \%$ en la no concertada), frente a un $47,5 \%$ que nunca antes lo había empleado con este fin $(58,5 \%$ de centros públicos, $35,7 \%$ de concertados y $25 \%$ de no concertados). El cálculo del "Coeficiente de Contingencia» y la Prueba "Chi-Cuadrado» ponen de manifiesto una asociación entre ambas variables (titularidad y uso de dispositivo con fines didácticos antes del confinamiento), aunque débil $(C=0,234)$, estadísticamente muy próxima a ser significativa $(p=0,057>0,05)$.

La Figura 1 representa las respuestas de los alumnos al ser cuestionados sobre el dispositivo empleado para dar clase durante el periodo de confinamiento. Destacamos el porcentaje de alumnos que indicó "no realizar esta tarea", siendo de un $50,9 \%$ en la titularidad pública y de un $31 \%$ en la concertada (ningún alumno de la no concertada señaló esta opción). En cuanto a los dispositivos más empleados con este fin extraemos que el porcentaje mayoritario en alumnos escolarizados en centros públicos señaló utilizar el smartphone (15,1\%), seguido del ordenador portátil (13,2\%) y la tablet (11,3\%). En la concertada la tablet se configura, por excelencia, como el dispositivo más elegido con un 28,6 \% de alumnos que confesó emplearlo para dar clases durante la cuarentena, seguido de un 23,8 \% que declaró recurrir al ordenador portátil y de un 9,5\% que empleó un ordenador de mesa. El $75 \%$ de los alumnos de centros no concertados se sirvió de la tablet y el $25 \%$ restante del ordenador portátil.

Cabe destacar que el $100 \%$ de nuestro alumnado ha estado recibiendo tareas para realizar durante el confinamiento. Así, el porcentaje más elevado de alumnos de centros públicos (un 30,2 \%) utilizó el smartphone con este fin. Los estudiantes de la concertada, por su parte, recurrieron, en su mayoría, al portátil (47,6\%) y la tablet (33,3\%), al igual que los alumnos de la no concertada (25\% al portátil y $75 \%$ a la tablet). El "Coeficiente de Contingencia» y la prueba "Chi-Cuadrado» (C $=0,386 ; \mathrm{p}=0,008<0,05)$ nos permiten deducir una asociación estadísticamente significativa entre la titularidad y los dispositivos empleados por los alumnos para recibir las tareas durante el confinamiento. 
PERCEPCIONES DE ALUMNOS Y DOCENTES DE $5 .^{\circ} \mathrm{Y} 60^{\circ}$ DE EDUCACIÓN PRIMARIA SOBRE LA MODALIDAD DE EDUCACIÓN A DISTANCIA IMPLANTADA TEMPORALMENTE EN ESPAÑA POR COVID-19

FIGURA 1

Relación entre la titularidad y el dispositivo empleado por los alumnos para dar clases, durante el confinamiento, a través de llamadas, videollamadas $\mathrm{u}$ otras aplicaciones habilitadas para ello

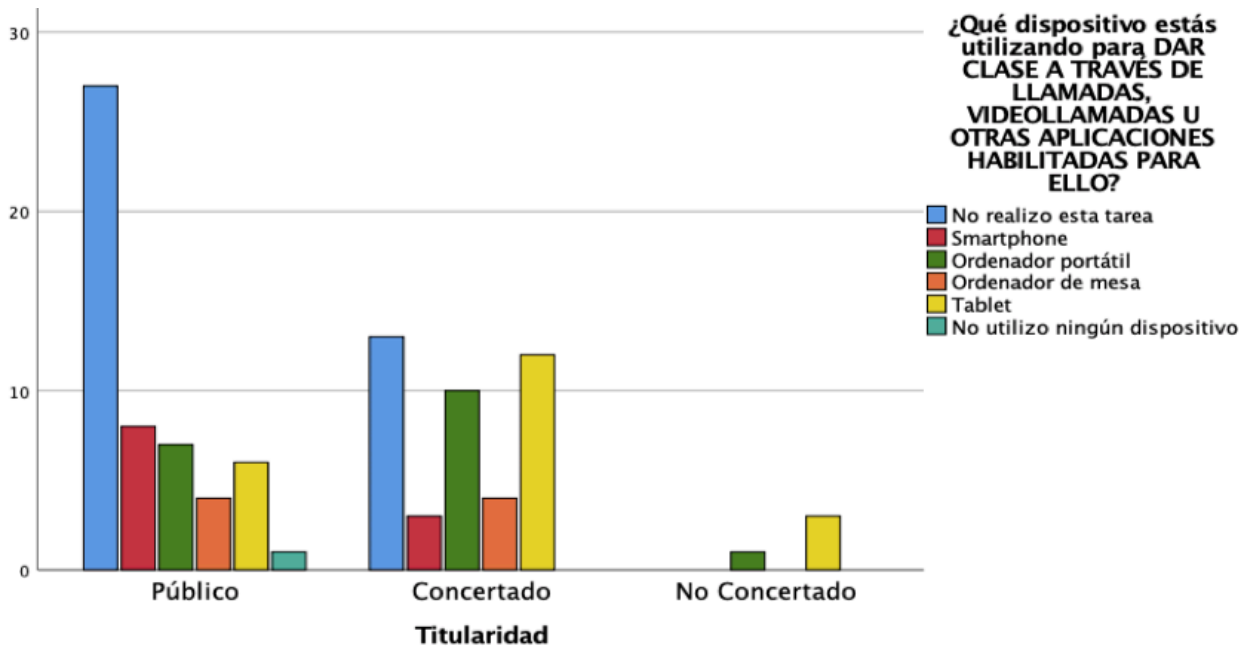

Fuente: Extracción de SPSS.

Con respecto a la entrega de tareas realizadas encontramos que la totalidad del porcentaje que no realizó esta tarea $(5,1 \%)$ recae en los alumnos de enseñanza publica, siendo el smartphone (con un $34 \%$ ) y el portátil (con un 22,6 \%) sus dispositivos más usados para esta función; en la concertada, los dispositivos más empleados con este fin fueron el portátil (con un 45,2 \%) y la tablet (con un $26,2 \%$ ), al igual que en la no concertada, con un $25 \%$ y un $75 \%$ respectivamente. De nuevo destacamos que el empleo del smartphone para esta tarea disminuye del $34 \%$ de la pública al 16,7 \% en la concertada y al $0 \%$ en la no concertada; así como el uso de la tablet, que aumenta del 18,9 \% en centros públicos al 26,2 \% en colegios concertados y al $75 \%$ en la no concertada. El «Coeficiente de Contingencia» y la prueba "Chi-Cuadrado» $(C=0,395 ; \mathrm{p}=0,050=0,05)$ nos permiten descubrir una asociación que podríamos clasificar como estadísticamente significativa entre ambas variables (titularidad y dispositivo empleado por los alumnos para entregar las tareas realizadas).

En cuanto a la recepción de notas, puntuaciones o valoraciones de tareas entregadas destacamos que un 20,8 \% de alumnos de centros públicos confesó no realizar esta tarea, junto a un $7,1 \%$ de la concertada. Tal como se observa en la Figura 2, la totalidad de alumnos de centros no concertados apuntó recibir sus tareas corregidas a través de tablet (25\%) o portátil (75\%). Nuestros alumnos de la pública seleccionaron, de nuevo, el smartphone por mayoría $(28,3 \%)$, seguido del 
PERCEPCIONES DE ALUMNOS Y DOCENTES DE $5 .^{\circ} \mathrm{Y} 6 .^{\circ}$ DE EDUCACIÓN PRIMARIA SOBRE LA MODALIDAD DE EDUCACIÓN A DISTANCIA IMPLANTADA TEMPORALMENTE EN ESPAÑA POR COVID-19

portátil (20,8 \%); y el alumnado de la concertada hizo referencia al portátil (47,6\%) y la tablet $(28,6 \%)$. En este caso, el "Coeficiente de Contingencia» y la prueba "ChiCuadrado" $(\mathrm{C}=0,408 ; \mathrm{p}=0,032<0,05)$ ponen de manifiesto la asociación estadísticamente significativa entre ambas variables (titularidad y dispositivo empleado por los alumnos para la recepción de notas, puntuaciones o valoraciones de tareas entregadas, durante el periodo de confinamiento).

FIGURA 2

Relación entre la titularidad y el dispositivo empleado por los alumnos, durante el confinamiento, para la recepción de notas, puntuaciones y valoraciones de tareas entregadas

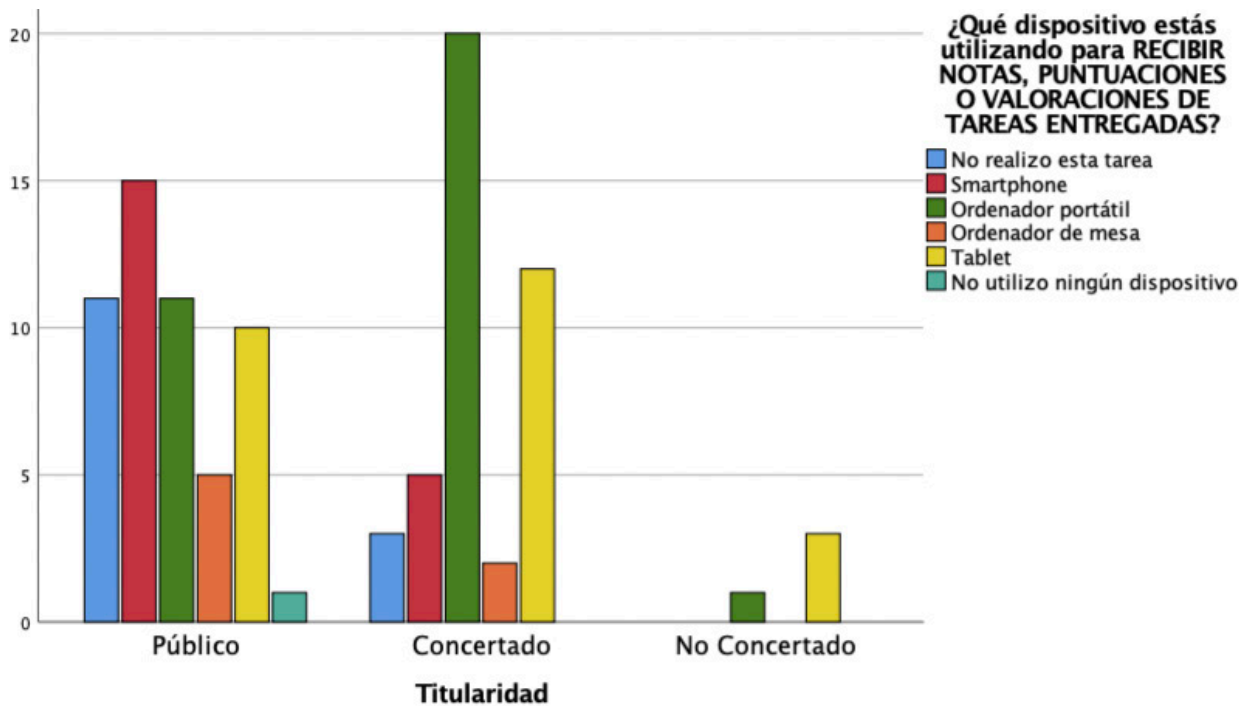

Fuente: Extracción de SPSS.

La búsqueda de información recalca lo ya observado y pone de manifiesto la asociación estadísticamente significativa entre las variables $(C=0,414 ; p=0,009<$ 0,05). El smartphone y el portátil fueron los dispositivos utilizados, por excelencia, por los alumnos de centros públicos para buscar información (con un 32,1 \% y un $22,6 \%$, respectivamente); el portátil y la tablet los más usados en la concertada (con un $45,2 \%$ y un $31 \%$, respectivamente) y en la no concertada (con un $25 \%$ y un $75 \%$, respectivamente).

Para la revisión del correo electrónico, de nuevo, los alumnos de centros públicos recurren al smartphone (35,8\%) y al ordenador portátil (22,6\%), y los alumnos de centros concertados al portátil (47,6 \%) y a la tablet $(23,8 \%)$, al igual que los de la no concertada, con un $25 \%$ que recurren al primero y un $75 \%$ que utilizan la segunda. Además, un 13,2 \% de la pública declaró no revisar el correo 
electrónico, porcentaje que desciende al 11,9 \% en la concertada y desaparece en la no concertada. El cálculo del "Coeficiente de Contingencia» y la prueba "ChiCuadrado» demuestran una asociación estadísticamente significativa $(C=0,430 ; \mathrm{p}=$ $0,013<0,05)$ entre la titularidad de los centros en los que los alumnos de nuestro estudio se encuentran escolarizados y la revisión que, durante el periodo del confinamiento, han hecho del correo electrónico.

Más de la mitad de los alumnos de centros públicos (52,8 \%) confesaron «no realizar exámenes» durante el periodo de confinamiento; porcentaje que se redujo a un $28,6 \%$ en colegios concertados y a un $25 \%$ en los no concertados. La prueba "Chi-Cuadrado» y el "Cálculo del "Coeficiente de Contingencia» reflejan la asociación estadísticamente significativa entre la titularidad y la realización de exámenes durante el confinamiento $(\mathrm{C}=0,465 ; \mathrm{p}=0,002<0,05)$. El ordenador portátil (con un $15,1 \%$ ) y el smartphone (con un 13,2\%) siguen siendo los dispositivos más usados por alumnos de centros públicos, y el portátil y la tablet (con un 45,2\% y un $19 \%$ respectivamente) en centros concertados. El $75 \%$ de los alumnos de la no concertada señaló preferir la tablet para realizar exámenes durante el confinamiento.

Ante la aparición de dudas durante la realización de las tareas escolares, el 58,6 \% de nuestros alumnos confesó solicitar la ayuda de algún familiar, un 35,4 \% afirmó poder preguntar al profesor/a ante la necesidad de aclaraciones y un 6,1\% indicó tratar de resolverlas por sí mismo. Se observa que el porcentaje mayoritario de alumnos de centros públicos $(69,8 \%$ ) señaló pedir ayuda a familiares para resolver dudas; en cambio, un $50 \%$ de los estudiantes de la concertada y un $75 \%$ de la no concertada apuntó la posibilidad de solicitar aclaraciones a sus docentes.

Destacamos, también, un porcentaje del 9,4\% de alumnos de la pública que tratan de resolver por sí mismos las dudas que les surgen, que disminuye al 2,4\% en colegios concertados y desaparece en los no concertados. Tras el cálculo del "Coeficiente de Contingencia» y la prueba "Chi-Cuadrado" se comprueba la asociación estadísticamente significativa $(C=0,334 ; p=0,014<0,05)$ entre ambas variables (titularidad y acciones de los alumnos ante la aparición de dudas).

El tiempo diario dedicado por los alumnos de colegios públicos que cumplimentaron nuestro cuestionario al estudio y las tareas escolares fue, en su mayoría, de entre dos y tres horas (un 39,6 \% de la totalidad de alumnos de dicha titularidad), seguidos por un porcentaje del $37,7 \%$ de estudiantes que dedicaba entre una y dos horas diarias durante el confinamiento a sus labores escolares. En el caso de la titularidad concertada, resalta un 47,6 \% que dedicó entre dos y tres horas diarias, seguido de un 33,3 \% que confesó emplear más de tres horas diarias en el estudio y realización de tareas. En la no concertada el 75 \% de los estudiantes indicó estudiar y realizar tareas durante más de tres horas. Subrayamos que un 5,7\% de los alumnos de enseñanza pública dedicó menos de una hora diaria durante el confinamiento al estudio, franja temporal que no fue señalada por ningún alumno de la concertada y de la no concertada. Una vez más, el cálculo del «Coeficiente de Contingencia y la prueba "Chi-Cuadrado" $(C=0,346 ; p=0,036<0,05)$ demuestran 
PERCEPCIONES DE ALUMNOS Y DOCENTES DE $5 .^{\circ} \mathrm{Y} 6 .^{\circ}$ DE EDUCACIÓN PRIMARIA SOBRE LA MODALIDAD DE EDUCACIÓN A DISTANCIA IMPLANTADA TEMPORALMENTE EN ESPAÑA POR COVID-19

la asociación estadísticamente significativa entre la titularidad y el tiempo medio diario dedicado por los alumnos de $5 .^{\circ}$ y $6 .^{\circ}$ al estudio y la realización de tareas escolares durante el confinamiento.

En relación a los recursos digitales utilizados por los alumnos de nuestro estudio para continuar con sus clases desde casa, predominan herramientas que permiten la realización de videollamadas, como es el caso de Google Meet, Zoom, Skype o Microsoft Teams. La herramienta Google Classroom se configura, por excelencia, como la más escogida por nuestros estudiantes para la recepción y entrega de actividades, seguida de las plataformas institucionales de los distintos colegios, los correos electrónicos, los blogs educativos y Google Drive.

Fueron escasos los alumnos que señalaron de forma específica una herramienta para la realización de exámenes o evaluaciones online, pues la gran mayoría hacía referencia a tareas o actividades "para corregir» a las que, con probabilidad, los docentes habían atribuido un carácter evaluable. No obstante, entre los recursos nombrados por nuestros alumnos para "examinarse online» se encuentran Socrative: Home o los formularios de "Google Forms".

El medio de contacto entre docentes-alumnos-familias es, por amplia mayoría, el correo electrónico (muchos vinculados a las plataformas educativas virtuales de los colegios), seguido de Google Classroom (únicamente un par de estudiantes indicó comunicarse con sus docentes a través de llamadas telefónicas); y la comunicación entre alumnos es a través de WhatsApp.

Y en cuanto a la valoración que los alumnos hacen del proceso de enseñanza vivido durante el confinamiento, indicamos que en la amplia mayoría de los casos las respuestas alternaban un aspecto positivo con uno negativo (aunque, aquellas en las que únicamente se plasmaba uno, este solía ser negativo):

Me gusta pasar más tiempo en casa pero echo de menos a mis compañeros y solo me cuesta hacer las cosas.

Lo que más son los vídeos explicativos y la comunicación con los profesores. Lo que menos no estar en el cole con compañeras y profes.

Me gusta porque tengo más tiempo, pero no me gusta una cosa: que cuando tengo una duda que mis padres no me pueden ayudar tengo que esperar a que al profesor de la asignatura le llegue el correo y mandarme otro con la respuesta de la duda.

Se observa que entre los puntos positivos nombrados y explicados por los alumnos destacan todos aquellos relacionados con la metodología innovadora, amena y "divertida" de la modalidad a distancia temporal, así como el uso didáctico de las tecnologías. Entre los aspectos negativos predominan la necesidad de contacto físico con los compañeros y la preferencia de clases presenciales para la resolución de dudas. 
PERCEPCIONES DE ALUMNOS Y DOCENTES DE $5 .^{\circ} \mathrm{Y} 6 .^{\circ}$ DE EDUCACIÓN PRIMARIA SOBRE LA MODALIDAD DE EDUCACIÓN A DISTANCIA IMPLANTADA TEMPORALMENTE EN ESPAÑA POR COVID-19

\subsection{Atendiendo a las percepciones de docentes de 5. ${ }^{\circ}$ y $6 .^{\circ}$ de Educación Primaria de centros públicos, concertados y no concertados de Alcalá de Henares}

Nuestra muestra de docentes se compone de un total de 31 participantes (profesores y profesoras de $5 .^{\circ}$ y $6 .^{\circ}$ de Educación Primaria escogidos al azar en la localidad de Alcalá de Henares). Se comprueba un porcentaje bastante igualado de maestras y maestros, ocupando las primeras un 51,6 \% del total de la muestra y los segundos un $48,4 \%$. Las edades de los profesionales que participaron en este estudio se encuentran entre los 25 y los 63 años (amplio rango de valores que explica el alto valor de la desviación), siendo la edad media de nuestra muestra completa de 40,45 años (y desviación típica 9,95).

Destacamos que el $100 \%$ de nuestros docentes $\left(61,3 \%\right.$ de $5 .^{\circ}$ de Primaria y $38,7 \%$ de $6 .^{\circ}$ curso) afirmó que durante el periodo de confinamiento continuó impartiendo clases desde casa y un notable 87 \% declaró no considerar a sus alumnos lo suficientemente preparados para el cambio experimentado en la modalidad de enseñanza.

En relación al punto de vista de los docentes sobre cómo ha sido la carga lectiva durante el periodo de confinamiento, percibimos una evidente inclinación hacia los extremos, representando el mayor porcentaje, 45,2 \%, a aquellos maestros que la valoraron como "superior", seguidos de un 35,5\% que, por el contrario, la calificaron como «inferior». La opción "se mantiene igual" fue escogida por un mínimo 19,4 \% de docentes. Estudiando la asociación entre la titularidad y la percepción de nuestros profesores y profesoras sobre la carga lectiva, a través del cálculo estadístico de asociación "Chi-Cuadrado" y el "Coeficiente de Contingencia» extraemos que el Coeficiente de Contingencia entre ambas variables nominales es $C=0,246$, asociación bastante débil y estadísticamente no significativa ( $p=0,735$ > 0,05); lo que nos permite deducir que, bajo la percepción de los maestros, no hay asociación estadísticamente significativa entre la titularidad del centro en el que los docentes participantes en el estudio imparten clase y la carga lectiva impuesta durante el periodo de confinamiento.

Para continuar desempeñando su labor docente desde casa, nuestros maestros y maestras emplearon diversos dispositivos digitales, siendo el ordenador portátil, con un $67,7 \%$, el más utilizado; seguido de la tableta digital, con un 19,4\%, y el ordenador de mesa, seleccionado por un 9,7 \% de los profesionales. El 3,2 \% restante declaró utilizar su smartphone para este fin, configurándose así como la opción menos escogida. La relación entre la edad de nuestros docentes y el dispositivo empleado para trabajar se percibe en la Figura 3, que refleja el uso de la tableta digital y el smartphone en aquellos maestros y maestras menores de 40 años y el empleo del ordenador de mesa en docentes de 45 años en adelante. 
PERCEPCIONES DE ALUMNOS Y DOCENTES DE $5 .^{\circ} \mathrm{Y} 6 .^{\circ}$ DE EDUCACIÓN PRIMARIA SOBRE LA MODALIDAD DE EDUCACIÓN A DISTANCIA IMPLANTADA TEMPORALMENTE EN ESPAÑA POR COVID-19

\section{FIGURA 3}

Relación entre la edad de los docentes y el dispositivo empleado para ejercer su labor durante el confinamiento

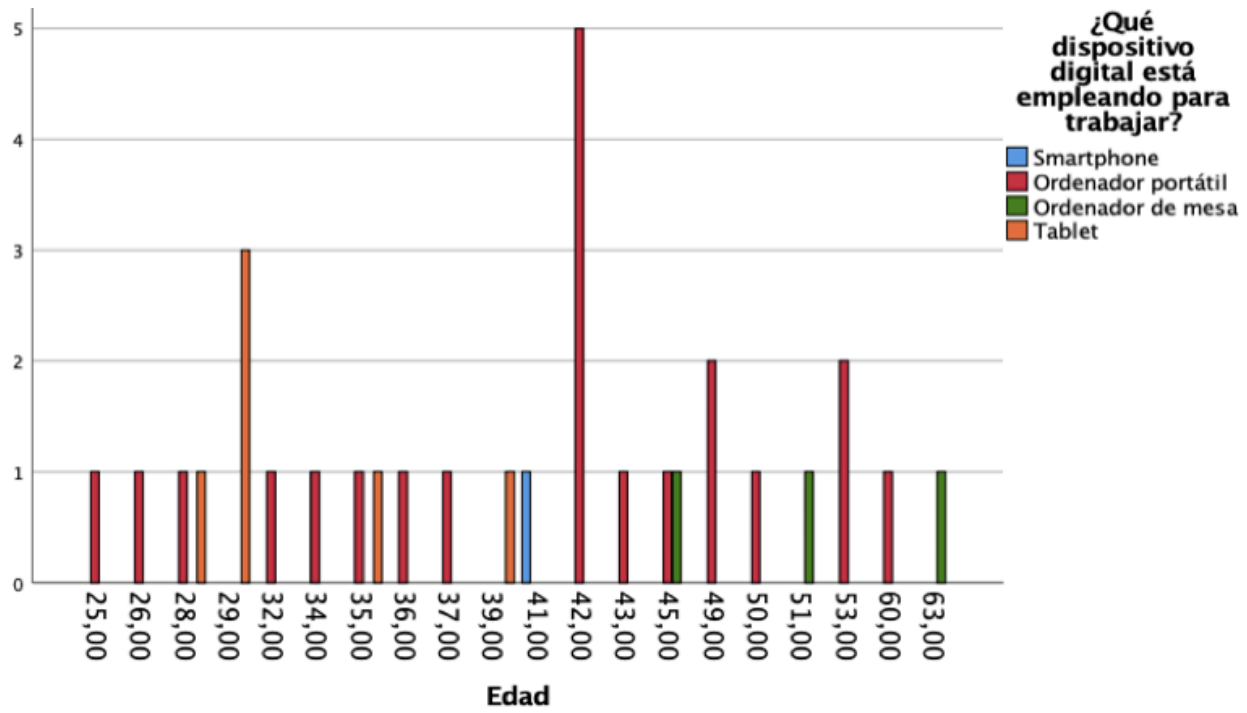

Fuente: Extracción de SPSS.

A la pregunta: ¿Sabe si sus alumnos disponen de un dispositivo digital propio o compartido para seguir con las clases desde casa? Un amplio 61,3\% de los profesores y profesoras respondió que sí, pues se habían asegurado de que sus estudiantes contaban con alguno previamente a planificar el desarrollo de las clases. No obstante, un destacado 32,2 \% declaró no estar seguro de ello y un mínimo 6,5 \% aseguró mantener el contacto con el alumnado a través de otros medios.

La selección de herramientas de trabajo virtuales para continuar con las clases durante el periodo de confinamiento se llevó a cabo, en un 54,8 \% de los casos, por los centros escolares, que facilitaron el acceso a nuevas plataformas o recursos; un $25,8 \%$ de los docentes señaló haber podido escoger aquellas que mejor se adaptaban a su situación, a su asignatura o a su grupo de alumnos; y el 19,4\% restante apuntó estar utilizando alguna plataforma que ya empleaba con anterioridad. La influencia de la titularidad en la selección de herramientas de trabajo se evidencia con el cálculo del "Coeficiente de Contingencia» y la prueba "ChiCuadrado", obteniendo una asociación considerable $(\mathrm{C}=0,544)$ y estadísticamente significativa $(\mathrm{p}=0,011<0,05)$ que nos lleva a aceptar la existencia de una relación entre ambas variables. Comprobamos que un abundante porcentaje de docentes de la enseñanza pública $(66,7 \%)$ ha podido escoger las herramientas que mejor se adaptaban a su situación (frente a un escaso 9,5\% de maestros de la concertada) 
y un mayoritario $71,4 \%$ de docentes en centros de titularidad concertada (100\% en la no concertada) señaló al colegio como facilitador de nuevas plataformas o recursos para continuar con la enseñanza durante el confinamiento. El porcentaje de profesores y profesoras que indicó utilizar con este fin una plataforma que ya empleaba con anterioridad a esta situación aumenta del 0,0 \% en la no concertada, a un $19,0 \%$ en la concertada y a un $22,2 \%$ en la pública.

En este estudio, los docentes tuvieron, además, la oportunidad de expresarse abiertamente compartiendo aspectos relacionados con el proceso formativo seguido (especificando plataformas educativas empleadas, herramientas virtuales o recursos didácticos...); percepciones sobre inconvenientes detectados durante la modalidad de enseñanza a distancia implantada temporalmente, e incluso sensaciones que reflejaran el apoyo sentido, la ayuda percibida o la valoración hacia su trabajo en esos días. Del análisis de sus respuestas extraemos la gran variedad de herramientas digitales empleadas por los docentes para hacer posible la continuación del proceso de enseñanza-aprendizaje desde casa, entre los que destacan: Google Classroom, Zoom o plataformas institucionales como instrumentos que facilitan la nueva metodología basada en la realización de clases virtuales, la transmisión de vídeos explicativos, la aclaración de conceptos, la preparación y corrección de tareas e incluso la realización de exámenes. Y entre los medios de contacto empleados por los docentes para mantener la comunicación con otros compañeros docentes, alumnos o familias de estos destacamos el email y las plataformas educativas (en algún caso se nombró el teléfono como vía de comunicación con familias). El estudio de las dificultades nos posibilita extraer la importancia de la cercanía docente-discente en los procesos de enseñanza-aprendizaje para comprobar las reacciones o impresiones de los alumnos en todo momento. Asimismo, parece sobresalir la falta de habilidades tecnológicas en los alumnos para el empleo de dispositivos con fines didácticos y la necesidad de medios en las familias para implantar una enseñanza de este tipo. Entre las dificultades relacionadas con el horario y el establecimiento de rutinas, los docentes destacan la organización que requiere una modalidad de enseñanza a distancia que depende, en gran medida, del grado de autonomía del alumnado. Las impresiones positivas percibidas por los profesionales de nuestro estudio sobre su labor docente están ligadas al apoyo de las familias y del centro y aquellas negativas tienen relación más directa con la sociedad a nivel general y el menosprecio generalizado, en ocasiones, del trabajo docente: «Siento el apoyo de las familias y del centro». "La respuesta social a nuestra labor ha sido de crítica destructiva».

4.3. Atendiendo a las vivencias y experiencias personales de tres maestros/as de $5^{\circ}$ y $6 .^{\circ}$ de Educación Primaria de centros públicos, concertados y no concertados de Alcalá de Henares

Las entrevistas individuales a cada uno de los tres profesores/as voluntarios para participar en nuestro estudio nos permitieron acercarnos de cerca a las 
PERCEPCIONES DE ALUMNOS Y DOCENTES DE $50^{\circ} \mathrm{Y} 60^{\circ}$ DE EDUCACIÓN PRIMARIA SOBRE LA MODALIDAD DE EDUCACIÓN A DISTANCIA IMPLANTADA TEMPORALMENTE EN ESPAÑA POR COVID-19

experiencias personales vividas por cada uno de ellos en el ejercicio de su labor docente durante el confinamiento, entendiendo sus miedos y compartiendo sus incertidumbres. Del análisis de las entrevistas extraemos que la adaptación a la nueva modalidad de aprendizaje generó malestar e incluso ansiedad en dos de nuestros profesionales. Además, el proceso de aprendizaje en la enseñanza telemática se apoyó en un avance de contenidos o temario y en una reprogramación que modificó, principalmente, la metodología y la evaluación; una metodología que recurrió a recursos digitales para posibilitar el aprendizaje desde casa (correo electrónico, plataforma institucional, «Microsoft Teams»...) y una evaluación llevada a cabo a través de la valoración de trabajos y tareas y de forma, principalmente, estimativa, siguiendo las recomendaciones del Gobierno. La comunicación docente-alumnos-familias, según nuestros tres profesionales, fue fluida y a través de diversos recursos virtuales (videoconferencias, plataformas, vía telefónica).

Nuestros docentes entrevistados apuntaron como dificultades detectadas en la enseñanza telemática el ritmo de aprendizaje, que se vio ralentizado afectando al trabajo de contenidos; la importancia de la disponibilidad de recursos para seguir con las clases desde casa (pues, aunque todos indicaron contar con un dispositivo propio desde el que trabajar, dejaron clara la flexibilidad ofrecida al alumnado entendiendo las circunstancias personales de cada familia); la complejidad de percibir el grado de comprensión de los alumnos de los contenidos trabajados (echando en falta el contacto directo y la explicación oral); y la escueta información que se les proporcionó, a nivel estatal, en todo momento.

Por último, y en relación a la competencia medial docente y digital discente, destacamos que todos nuestros profesionales habían recibido, por cuenta propia o en su mismo centro, formación en el uso de los medios digitales (anterior al confinamiento) y se aprecia cierta controversia entre la maestra que afirmó la desarrollada capacidad de su alumnado en el manejo de las TIC, el docente que opinó que los estudiantes estaban capacitados para su uso y manejo (aunque señaló que en su centro, por determinación del equipo directivo, fueron las familias quienes "mediaron» en el proceso educativo entre el docente y los alumnos) y la profesora que aseguró percibir escasez de habilidades tecnológicas en sus alumnos con respecto al uso y manejo de las plataformas virtuales y programas empleados durante la modalidad de educación a distancia.

\section{CONCLUSIONES}

Atendiendo a las percepciones de los alumnos comprobamos que el $100 \%$ de los mismos afirmó disponer de conexión a Red Wifi y un 85 \% declaró contar con un dispositivo propio desde el que realizar las tareas, lo que coincide con las declaraciones de Serrano y Martínez (2003) sobre la cada vez menos evidente brecha digital dado que los dispositivos móviles permiten el acceso a las TIC sin suponer un coste muy elevado. 

DE EDUCACIÓN A DISTANCIA IMPLANTADA TEMPORALMENTE EN ESPAÑA POR COVID-19

Recordamos que Camacho y Esteve (2018) constataban con su estudio la actitud favorable de los alumnos hacia las TIC y la percepción de estos (sobre sí mismos) como competentes en el uso de la tecnología móvil, punto que podemos verificar con nuestro estudio al haber confirmado un $63,8 \%$ de los estudiantes de $5 .^{\circ}$ y un $75 \%$ de los de $6 .^{\circ}$ su facilidad para el manejo de los dispositivos digitales.

Un amplio 47,5 \% de nuestro alumnado confesó no haber empleado, anteriormente al confinamiento, los dispositivos digitales para realizar las tareas escolares, lo que coincide con el estudio llevado a cabo por Empantallados y GAD3 y sus resultados sobre el incremento (en un $76 \%$ ) de los más pequeños en el uso diario de las pantallas en el confinamiento. De nuestro estudio extraemos, además, la asociación significativa entre la titularidad de los centros en los que los alumnos se encontraban matriculados y los dispositivos empleados por estos para recibir tareas para realizar, así como notas o valoraciones, buscar información o revisar el correo electrónico, siendo el smartphone el dispositivo empleado por excelencia por estudiantes de la titularidad pública, el portátil por la concertada y la tablet por la no concertada. Entre los recursos digitales empleados por los alumnos participantes en el estudio para continuar con las clases desde casa predominan: Google Classroom, herramientas que permiten la realización de videollamadas (Google Meet, Zoom, Skype o Microsoft Teams son las más señaladas), las plataformas institucionales de los centros y los correos electrónicos.

La percepción de los docentes nos permite constatar la diversidad de opiniones con respecto a la carga lectiva, siendo esta valorada por un 45,2 \% de maestros como «superior» y un 35,5\% como «inferior». Se comprueba que el dispositivo más recurrido para el trabajo docente es el ordenador portátil (por un 67,7\% de los maestros y maestras de nuestro estudio), aunque a su vez se percibe un uso del smartphone y la tablet superior en docentes menores de 40 años (posiblemente por tratarse de dispositivos más actuales) y del ordenador de mesa en aquellos de 45 años en adelante. Extraemos, también, que un 66,7 \% de los docentes de la pública manifestaron haber podido elegir aquellas herramientas que mejor se adaptaban a su situación, frente al porcentaje mayoritario de la concertada $(71,4 \%)$ que señaló al centro como facilitador de las plataformas o recursos para continuar con las clases desde casa.

Las entrevistas personales con nuestros docentes permitieron percibir la angustia generalizada durante el proceso de adaptación, debido en gran parte a la incertidumbre y al "no saber cómo actuar», y destacamos como dificultades detectadas por los maestros y maestras de nuestro estudio en la enseñanza durante el confinamiento la ralentización del ritmo de trabajo (muy posiblemente debido a la falta de metodologías didácticas digitales que favorezcan la equidad en este aspecto).

Este estudio, por tanto, ha permitido confirmar nuestra hipótesis al comprobar que la modalidad de enseñanza telemática implantada en España durante el confinamiento ha incrementado el uso de dispositivos digitales y herramientas virtuales permitiendo a alumnos y docentes continuar con los procesos de enseñanzaaprendizaje desde casa. Como limitaciones se señalan la imposibilidad de realizar 
PERCEPCIONES DE ALUMNOS Y DOCENTES DE $5 .^{\circ} \mathrm{Y} 6 .^{\circ}$ DE EDUCACIÓN PRIMARIA SOBRE LA MODALIDAD DE EDUCACIÓN A DISTANCIA IMPLANTADA TEMPORALMENTE EN ESPAÑA POR COVID-19

las entrevistas de manera presencial y la de poder hacer llegar en formato físico a un mayor número de estudiantes y docentes los cuestionarios objeto de análisis (lo que nos hubiera permitido obtener una muestra más amplia). Se valora la posibilidad de ampliar el estudio analizando el desarrollo de la competencia digital de los alumnos tras la vuelta a clase después del confinamiento.

\section{REFERENCIAS BIBLIOGRÁFICAS}

Aguilar, M. (2012). Aprendizaje y Tecnologías de Información y Comunicación: Hacia nuevos escenarios educativos. Revista Latinoamericana de Ciencias Sociales, Niñez y Juventud, 10 (2), 801-811.

Alonso, M. H., González, J. E. y Muñoz, A. B. (2016). Ventajas e inconvenientes del uso de dispositivos electrónicos en el aula: percepción de los estudiantes de grados en comunicación. Revista de Comunicación de la SEECI, 20 (41), 136-154.

Area, M., Alonso, C., Correa, J. M., Moral, M. E., Pablos, J., Paredes, J., Peirats, J., Sanabria, A. L., San Martín, A. y Valverde, J. (2014). Las políticas educativas TIC en España después del Programa Escuela 2.0: las tendencias que emergen. Revista Latinoamericana de Tecnología Educativa, 13 (2), 11-33.

Cabero, J. (1998). Impacto de las nuevas tecnologías de la información y la comunicación en las organizaciones educativas. En M. Lorenzo y otros (Coords.), Enfoques en la organización y dirección de instituciones educativas formales y no formales. Actas de las II Jornadas Andaluzas sobre Organización y Dirección de Centros Educativos (pp. 197-206). Granada: Grupo Editorial Universitario (GEU Editorial).

Calero, C. (2019). La llegada de las nuevas tecnologías a la educación y sus implicaciones. International Journal of New Education, 2 (2).

Camacho, M. y Esteve, F. M. (2018). El uso de las tabletas y su impacto en el aprendizaje. Una investigación nacional en centros de Educación Primaria. Revista de Educación, 379, 170-191.

Castells, M. (Ed.). (2006). La sociedad red: una visión global. Madrid: Alianza Editorial.

Castells, M. y Cardoso, G. (Eds.). (2005). The Network Society From Knowledge to Policy. Washington, DC: Johns Hopkins Center for Transatlantic Relations.

Díaz-Barriga, A. (2013). TIC en el trabajo del aula. Impacto en la planeación didáctica. Revista Iberoamericana de Educación Superior, 10 (4), 3-21.

Ecoaula.es (2020, 13 de mayo). Los padres españoles confían en la tecnología para el desarrollo profesional y educativo de sus hijos. El Economista.es.

Feito, R. (2020). Este es el fin de la escuela tal y como la conocemos. Unas reflexiones en tiempo de confinamiento. Revista de Sociología de la Educación-RASE, 13 (2), Especial Covid-19, 156-163.

Fernández, L. (2016). El uso didáctico y metodológico de las Tabletas Digitales en aulas de Educación Primaria y Secundaria de Cataluña. Pixel-Bit. Revista de Medios y Educación, (48), 9-25.

Fernández, P. y Vergara, D. (2020). La digitalización del sistema educativo como consecuencia del Covid-19. Magisterio.

Fombona, J. y Pascual, M. A. (2013). Beneficios del m-learning en la Educación Superior. Educatio Siglo XXI, 31 (2), 211-234. 

DE EDUCACIÓN A DISTANCIA IMPLANTADA TEMPORALMENTE EN ESPAÑA POR COVID-19

Hamodi, C. y Bermejo, M. I. (2014). Impacto educativo de las tabletas digitales: estudio de caso de una experiencia innovadora. Actualidades Pedagógicas, (63), 157-179.

Hembrooke, H. y Gay, G. (2003). The Laptop and the Lecture: The effects of Multitasking in Learning Environments. Journal of Computing in Higher Education, 15 (1).

Hernández, M. (2020, 6 de febrero). El uso del móvil en las aulas: educar en el mundo real. La Vanguardia.

Instituto Nacional de Estadística. (2019). Encuesta sobre Equipamiento y Uso de Tecnologías de Información y Comunicación en los Hogares. Notas de prensa.

Instituto Nacional de Tecnologías Educativas y de Formación del Profesorado. (2017). Una breve historia de las TIC Educativas en España. Ministerio de Educación, Cultura y Deporte.

Lozano, R. (2011). De las TIC a las TAC: tecnologías del aprendizaje y del conocimiento. Anuario ThinkEPI, 5, 45-47.

Marés, L. (2012). Tablets en Educación. Oportunidades y desafíos en políticas uno a uno. Red Latinoamericana portales educativos.

Martí, M. C., D’Agostino, M. J., Veiga, J. y Sanz-Valero, J. (2008). Alfabetización Digital: un peldaño hacia la sociedad de la información. Medicina y Seguridad del Trabajo, 54 (210), 11-15.

Ministerio de Ciencia y Tecnología. (s. f.). La Sociedad de la Información en el siglo XXI: un requisito para el desarrollo. Buenas prácticas y lecciones aprendidas.

Organización de las Naciones Unidas para la Educación, la Ciencia y la Cultura. (2013). Directrices de la UNESCO para las políticas de aprendizaje móvil.

Organización de las Naciones Unidas para la Educación, la Ciencia y la Cultura. (2020). Propuestas de la UNESCO para garantizar la educación online durante la pandemia. Educaweb.

Peralta, L. (2020, 15 de abril). José Antonio Marina: «Dar aprobado general, como piensa hacer Italia, es malo, una falsa solución». ABC. Educación.

Pérez, S. (2010). La importancia de las TICs en la escuela. Temas para la educación. Revista Digital para Profesionales de la Enseñanza.

Plaza, J. A. (2020, 24 de abril). Confinamiento y tecnología: digitalizados a marchas forzadas. Revista Retina. El País.Com.

Pulido, D. C., Nájar, O. y Guesguán, L. G. (2015). Vivamos la innovación de la inclusión de dispositivos móviles en la Educación. Praxis \& Saber, 7 (14).

Sáez, B., Viñegla, M. P., López, M. M., López, J. M. y García, M. (2011). Smartphones: una potente herramienta para la docencia. En IX Jornadas Internacionales de Innovación Universitaria. Retos y oportunidades del desarrollo de los nuevos títulos en educación superior. Universidad Politécnica de Madrid.

Sáez, J. M., Sevillano, M. L. y Vázquez, E. (2019). El uso académico del ordenador portátil y del Smartphone en estudiantes universitarios españoles e iberoamericanos. Education in the Knowledge Society, 20.

Salinas, J. (2002). Modelos flexibles como respuesta de las universidades a la sociedad de la información. Acción Pedagógica, 11 (1), 4-13.

Santiago, R., Amo, D. y Díez, A. (2014). ¿Pueden las aplicaciones educativas de los dispositivos móviles ayudar al desarrollo de las inteligencias múltiples? Revista Electrónica de Tecnología Educativa, (47), 1-10.

Servimedia (2020, 22 de mayo). La mitad de las familias compra dispositivos durante el confinamiento para la educación online, el teletrabajo o el ocio digital. El Economista.es. 
PERCEPCIONES DE ALUMNOS Y DOCENTES DE $5 .^{\circ} \mathrm{Y} 6 .^{\circ}$ DE EDUCACIÓN PRIMARIA SOBRE LA MODALIDAD DE EDUCACIÓN A DISTANCIA IMPLANTADA TEMPORALMENTE EN ESPAÑA POR COVID-19

Severin, E. (2014). Tecnologías de la Información y la Comunicación, TIC, para el aprendizaje. Apuntes Educación y Desarrollo Post-2015. Organización de las Naciones Unidas, para la Educación, la Ciencia y la Cultura, 3.

Sevillano, M. L. (2008). Nuevas tecnologías en educación social. Madrid: McGraw- Hill/Interamericana de España, S. A. U.

Sevillano, M. L. y Vázquez-Cano, E. (2015). Modelos de investigación en contextos ubicuos y móviles en Educación Superior. Madrid: McGraw-Hill/Interamericana de España, S. A. U.

Sevillano, M. L. y Vázquez-Cano, E. (2019). Dispositivos digitales móviles en Educación. El aprendizaje ubicuo. Madrid: Narcea, S. A. de ediciones.

Silva, A. C. y Martínez, D. G. (2017). Influencia del Smartphone en los procesos de aprendizaje y enseñanza. Suma de Negocios, 8 (17), 11-18.

Suárez, N. y Custodio, J. (2014). Evolución de las tecnologías de información y comunicación en el proceso de enseñanza-aprendizaje. Revista Vinculos, 11 (1), 209-220.

Suárez, C., Lloret, C. y Mengual, S. (2016). Percepción sobre la transformación digital del aula a través de tabletas: un estudio en el contexto español. Comunicar. Revista Científica de Educomunicación, 24 (49), 81-89.

Tarabini, A. (2020). ¿Para qué sirve la escuela? Reflexiones sociológicas en tiempos de pandemia global. Revista de Sociología de la Educación-RASE, 13 (2), Especial Covid-19, $145-155$.

Tragacetet, M. (2020, 9 de marzo). Madrid suspende las clases a 1,5 millones de alumnos tras doblarse en un día la cifra de afectados de coronavirus. La Vanguardia.

Uc, N. A., López, J. L. y Aguilar, M. C. (2017). Uso de la Laptop y su relación con el rendimiento académico de estudiantes de Bachillerato (pp. 1-10). Congreso Nacional de Investigación Educativa. San Luis Potosí. México. 
